\title{
High Precision Analysis of Stress Concentration in Girder Structure of Casting Crane
}

\author{
Qin Yixiao ${ }^{1}$, Jiang $\mathbf{J i}^{1}$, Yang Haiming ${ }^{1,2}$ \\ ${ }^{1}$ Department of Mechanical Engineering, Taiyuan University of Science and Technology, Taiyuan, China \\ ${ }^{2}$ Department of Mechanical Engineering, Taian Vocational Technology College, Taian, China
}

Email address:

qyx819@163.com (Qin Yixiao), 734363840@qq.com (Jiang Ji)

To cite this article:

Qin Yixiao, Jiang Ji, Yang Haiming. High Precision Analysis of Stress Concentration in Girder Structure of Casting Crane. International Journal of Science and Qualitative Analysis. Vol. 2, No. 2, 2016, pp. 14-18. doi: 10.11648/j.ijsqa.20160202.11

Received: July 12, 2016; Accepted: July 25, 2016; Published: August 26, 2016

\begin{abstract}
The stress concentration is easily occurred in the key local position of the main girder structure of casting crane with heavy load. The sub model method has the advantages of high efficiency and high precision in solving the complicated stress and strain problems of the local structure. Based on the result of finite element model analysis of whole bridge girder, the sub model technology is used to cut the local model of the main beam and partition more refined finite element meshes. The cutting boundary displacement of the whole bridge girder model is regarded as the forced displacement load that is applied on the boundary of the local model. The results that are closer to the engineering practical calculation can be obtained by analyzing and solving. The engineering analysis method in this paper can provide reference for product design.
\end{abstract}

Keywords: Stress Concentration, Sub Model, High-Precision, Analysis

\section{Introduction}

The casting crane has a large number of load spectrum, frequent use and poor working conditions. The bridge is under heavy load when lifting operations [1]. Especially when blending the molten steel, the main bridge girder structure is affected by the flame baking. Its safety performance deserves attention [2]. When the bridge girder is impacted by the frequent external load, the fatigue damage is easily occurred in the welds connecting with the transverse bulkhead and upper wing panel and the top of the web. The welds between the upper wing panel and the web and the welds between the transverse bulkhead and the web tension zone also have the same situation [3]. In order to ensure the safe operation of the crane and efficient work normally, it is necessary for the crane metal structure to carry through high precision finite element analysis and make sure the maximum stress and stress distribution in the work that may occur $[4,5]$.

\section{High Precision Analysis of Local Stress Concentration Area in Casting Crane Girder Structure}

\subsection{Sub Model Method}

The local structure of the system with the same characteristics and properties is called the sub structure. because the structure has a variety of geometric repeatability, it can be divided into multi-level sub structure. Super-element is a kind of generalized specific unit. In practical application, it can be produced according to the need, the produced super-element is actually a sub structure that retracts internal node degree of freedom [6]. It is shown as the degree of freedom of the nodes that only have a connection to the outside, the purpose of building a super-element is to reduce the amount of computation, especially in the complex calculation process which needs many iterations (such as contact problem, nonlinear analysis, etc.), it can fully show its superiority[7]. Due to the use of the super-element, we can greatly reduce the computation time of each generation of the stiffness matrix, also reduces the computation scale at the 
same time, So as to obtain a higher computational efficiency.

For an actual structure, If there is the following stiffness equation:

$$
\left[\begin{array}{cc}
K_{m m} & K_{m s} \\
K_{s m} & K_{s s}
\end{array}\right]\left[\begin{array}{c}
q_{m} \\
q_{s}
\end{array}\right]=\left[\begin{array}{c}
P_{m} \\
P_{s}
\end{array}\right]
$$

$q_{m}$ is node displacement for the master node, also named master DOF, $q_{s}$ is node displacement for the slave node. Generally speaking, the slave node is regarded as an internal node, after the condensation of the slave node displacement $q_{s}$, we can obtain condensation equation:

$$
\begin{gathered}
\bar{K} q_{m}=\bar{P} \\
\bar{K}=K_{m m}-K_{m s} K_{s s}^{-1} K_{s m} \\
\bar{P}=P_{m}-K_{m s} K_{s s}^{-1} P_{s}
\end{gathered}
$$

The equation (2) represents the element stiffness equation of the super element. $\bar{K}$ is the stiffness matrix of the super element, $\bar{P}$ is the load node array for the super-element. Figure 1 shows a schematic of the application of the super-element.

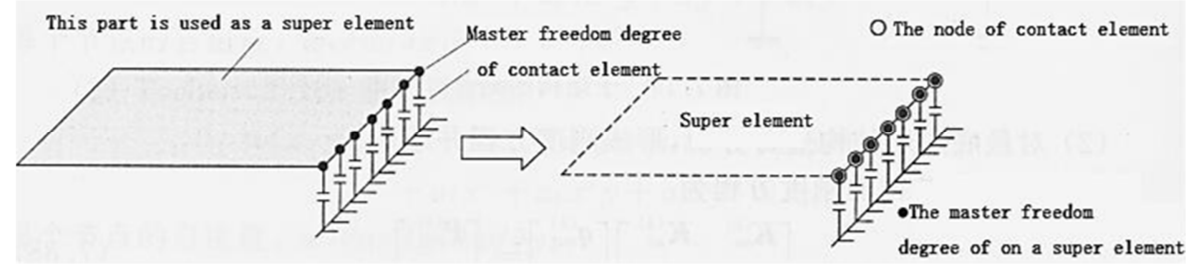

Fig. 1. Sub structural classification.

The sub model provides a new idea of design and analysis is that under the condition of the integral structure design conducts a design of local structure of multi scheme and increases the analysis accuracy of the stress distribution law of the whole structure, at the same time reduces computational effort and saves computer resources. The analytical accuracy obtained by the sub model analysis is higher than the whole model. Through the fine finite element calculation of the main beam local structure, the results of the overall model of the main beam, the actual test results, the results of the local structure sub model analysis are compared with the results of the same location data, the way is paved for the improvement of the local structure of the main beam in the future.

\subsection{Analysis of Local Stress Concentration Area in Casting Crane Girder}

The structure form of welding is adopted in the main beam of the casting crane. Lower wing panel is the most critical and dangerous part of the main beam structure. Once the lower wing panel weld appears a serious fatigue crack, it is likely to lead to safety accidents. In order to reduce the shear stress on the cross - end web plate and the bending plate, section form of $\mathrm{T}$ type steel and composite web plate is used in large tonnage casting crane aimed to improve the stress distribution of the welding seam. However, it increases the complexity of the cross-end structure of the main beam. The traditional calculation can not be applied to calculate accurately. In the local stress concentration area of the main beam structure, two representative hazardous areas are selected. One is the lower wing panel weld, the other is the main beam cross - end bending plate and the web weld.

Based on the crane partial rail box half bridge frame main beam overall finite element analysis results. A more accurate finite element analysis of the lower wing panel weld comes true by using sub model technique [8]. The finite element mesh of the sub model is shown in Figure 2 by selecting the lower part of the web and lower wing panel and setting up sub model and using the command stream generating sub model.

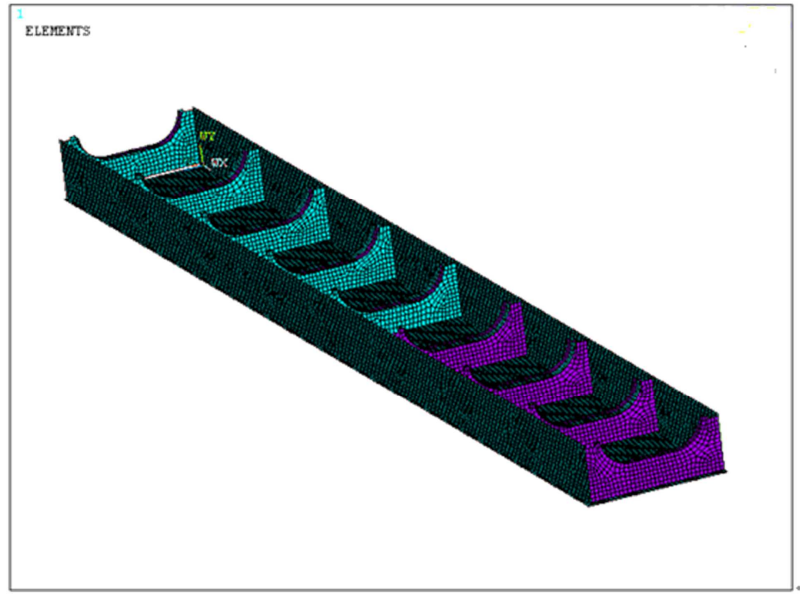

Fig. 2. The finite element meshes of sub model in mid-span lower wing panel.

Parts of the order are as follows [9-11]:

/CLEAR: ! Clear database

/FILNAME, SUBMOD: ! Define sub model file name /PREP7: ! Enter the pre processor

$\ldots:$ ! Generate sub model ( the overall model of bridge girder) CSYS, 0: ! Delete content except the overall model of bridge girder

...: ! (Omit part of the program)

ALLSEL: ! Grid encryption section

LESIZE, ALL, WGCC: ! Define unit length

MSHAPE, 0, 3D: ! Define the cell shape as the 3 dimensional 4 - shape element

MSHKEY, 0: !define the way that divide mesh is free mesh AMESH, ALL: ! Divide mesh

NSLL, S, 1: ! Write the cut edge node to the file SUBMOD. 
NODE

NWRITE

ALLSEL: ! Store integrated sub model database file SUBMOD. DB

SAVE

FINISH

RESUME, COARSE, DB: ! Read the whole bridge girder model database file COARSE. DB

/POST1: ! enter the post processor

FILE, COARSE, RST: ! Read the whole bridge girder modal database file

SET, 1

CBDOF: ! from SUBMOD. DB read the cut edge node and write command D to SUBMOD. CBDO

RESUME: ! Read the sub modal database SUBMOD. DB

/SOLU: ! Enter solver

/INPUT, SUBMOD, CBDO: ! Cut boundary freedom degree

SOLVE: ! Solve

\section{Results and Discussion}

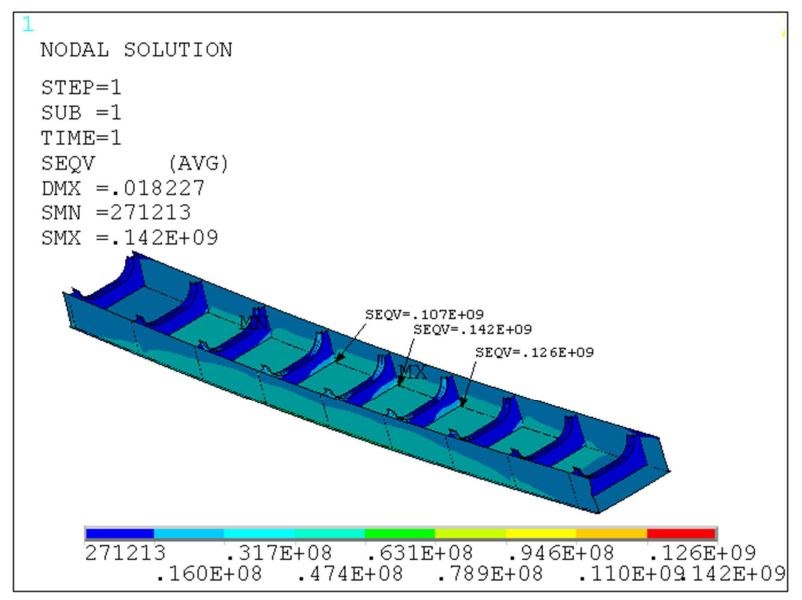

Fig. 3. The stress nephogram of whole model in lower wing panel.

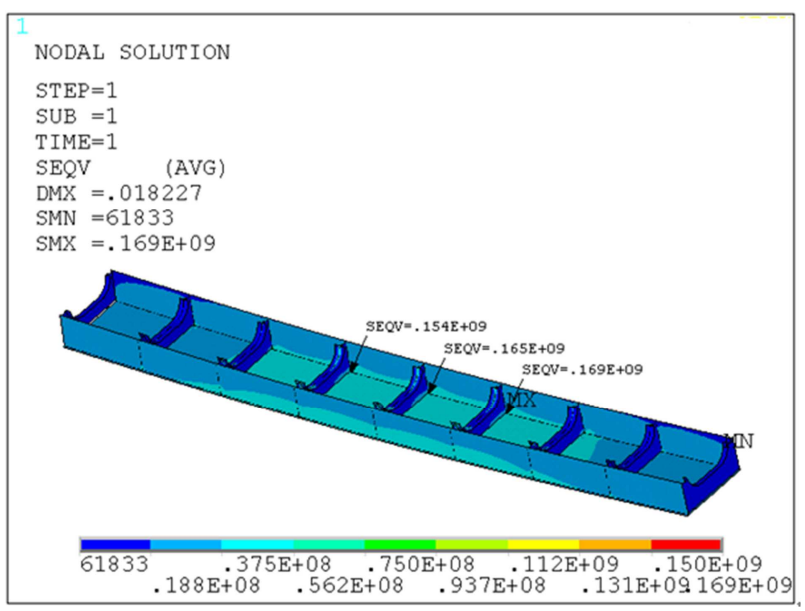

Fig. 4. The stress nephogram of sub model in lower wing panel.

When the middle diaphragm loads, the whole lower wing panel modal stress nephogram calculated by finite element analysis is shown in Figure 3. Stress values in three positions of the mid-span lower wing panel and transverse bulkhead in the whole modal respectively are $107 \mathrm{MPa}, 142 \mathrm{MPa}, 126 \mathrm{MPa}$, and the lower wing panel weld fatigue allowable stress is 184.2MPa.

When the middle diaphragm loads, the lower wing panel sub modal stress nephogram calculated by finite element analysis is shown in Figure 4. Stress values in three position of the mid-span lower wing panel and transverse bulkhead in the sub modal respectively are $154 \mathrm{MPa}, 165 \mathrm{MPa}, 169 \mathrm{MPa}$, and the lower wing panel weld fatigue allowable stress is 184.2MPa.

Comparing the sub modal and the whole modal, the stress amplitude of the three positions also reach $43.9 \%, 16.2 \%$ and $34 \%$ respectively in this picture. It is obvious that the actual bending stress of the mid-span lower wing panel is very large, the whole finite element analysis results of the main girder are smaller than the sub model analysis, and the stress distribution of the lower wing panel weld can not be more accurate.

Based on the results of the main girder finite element analysis, a more accurate finite element analysis for the complex structure of the cross-end structure is accomplished by using the sub model technique so that get shear stress close to the engineering practice of the main girder span web and bending plate position.

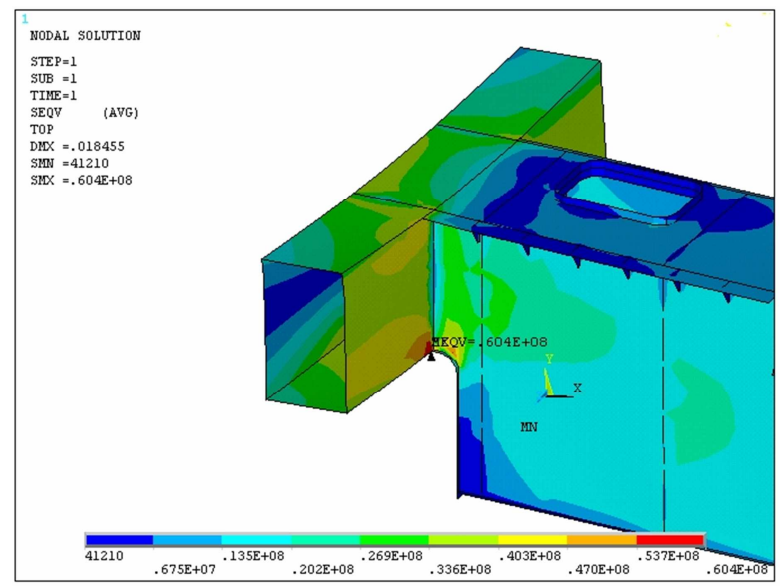

Fig. 5. The stress nephogram of whole model in cross-end (the main web side).

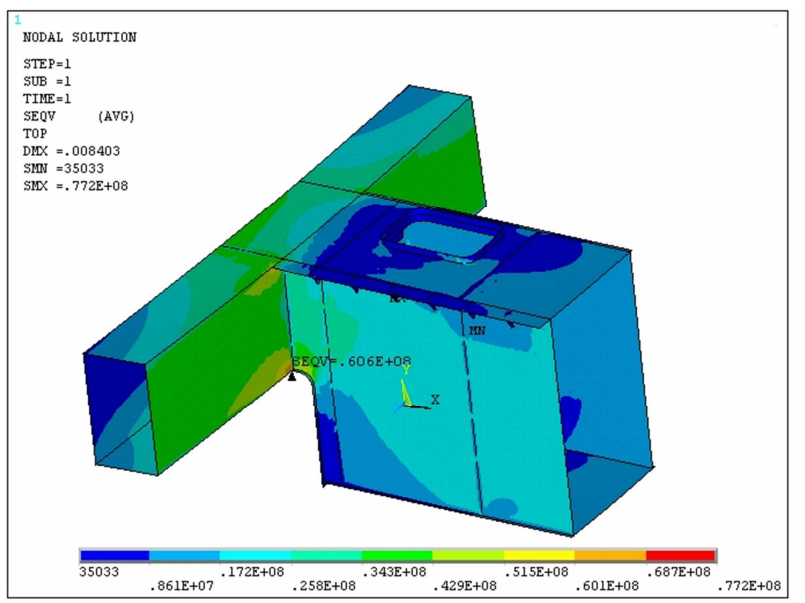

Fig. 6. The stress nephogram of sub model in cross-end (the main web side). 
From Figure 5, we find that the equivalent maximum stress of the node is $60.4 \mathrm{MPa}$, the minimum stress is $41210 \mathrm{~Pa}$, and the maximum displacement is $18.455 \mathrm{~mm}$ in the cross - end whole model.

From figure 6 , we find that equivalent maximum stress solution of the node is $77.2 \mathrm{MPa}$ in cross-end sub modal. However, the maximum stress of the model is $60.6 \mathrm{MPa}$, the minimum stress is $35033 \mathrm{~Pa}$, the maximum displacement is $8.403 \mathrm{~mm}$.

The finite element analysis stress nephogram of the whole model and the sub model are very similar in the main girder span web and bending plate position, the maximum shear stress is about $60.6 \mathrm{MPa}$. The results show that the stress calculation of the whole model is very precise and the data is worth learning. It is also proved that the welding stress in the cross section of the large tonnage casting crane can be improved obviously after the section use the T type steel and the composite web plate.

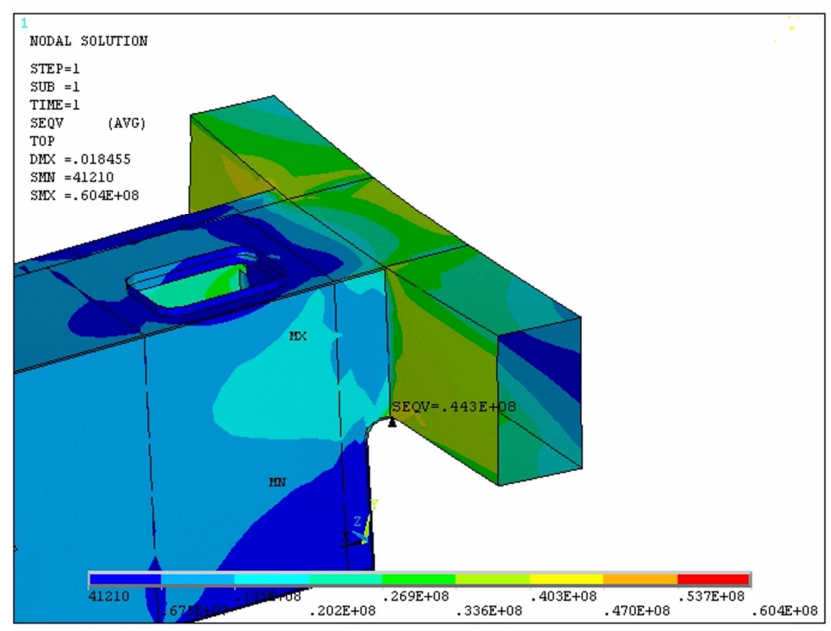

Fig. 7. The stress nephogram of whole model in cross-end (Vice-web side).

From figure 7, we find that the equivalent maximum stress solution of the node is $60.4 \mathrm{MPa}$ in the cross-end whole modal, the maximum stress of the model is $44.3 \mathrm{MPa}$, the minimum stress is $41210 \mathrm{~Pa}$, and the maximum displacement is $18.455 \mathrm{~mm}$.

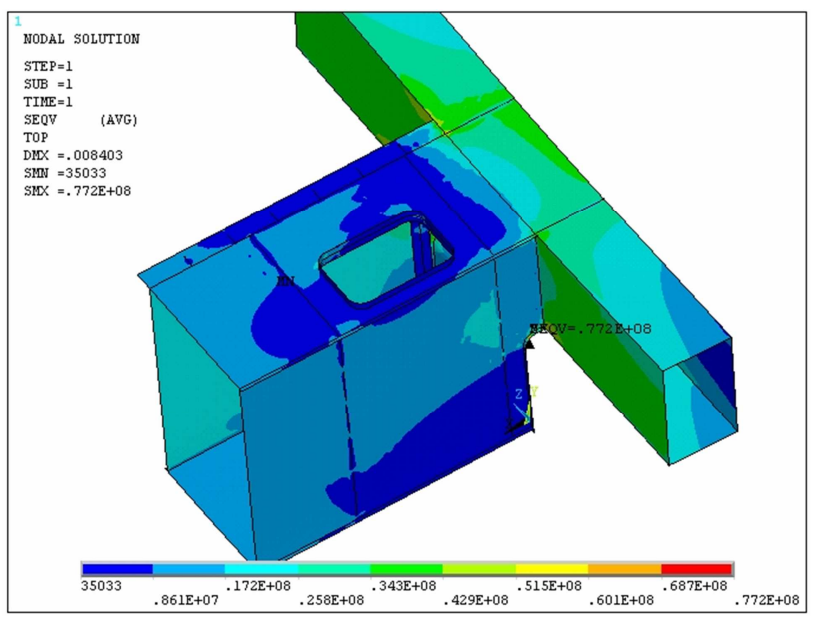

Fig. 8. The stress nephogram of sub model in cross-end (Vice-web side).
From figure 8, we find that equivalent maximum stress solution of the node is $77.2 \mathrm{MPa}$ in the cross-end sub modal, the maximum stress in the model is $77.2 \mathrm{MPa}$, the minimum stress is $35033 \mathrm{~Pa}$, and the maximum displacement is $8.403 \mathrm{~mm}$.

In the finite element analysis stress nephogram of the whole model and the sub model where the span side plate and the bending plate position of the cross-end main beam (the section of the main girder), the results of maximum shear stress are relatively large, but the maximum shear stress is within the allowable shear stress (100 MPa), which can satisfy the requirements of design and application. And the main web stress is similar basically with the secondary web, there is no big difference, the structural is stable.

\section{Conclusion}

The use of sub model technology can effectively improve the analysis accuracy of the casting crane local structure. According to the results of the whole model of the main girder, different sub models are created for the many local structures of the main beam, which can effectively improve the accuracy of the calculation results in the local stress concentration area of the main beam.

The accurate finite element analysis of complex structures can be realized by using the high precision method, not only improve the design efficiency of bridge structure but also evaluate and make better of rationality of the local structure of the bridge structure. So the bridge structure design obtains optimization, comes true accurate and reasonable design of crane structure.

To ensure that the sub model is constructed under the condition that the cut boundary is far from the stress concentration area, a high-precision analysis is presented for two dangerous parts of the stress distribution rules on the casting crane girder and help the designers to understand the situation of the whole girder strength reserve, so that the local structure was optimized and improved in the process of product design and manufacturing, the method can be helpful for local structure where have the dramatic changes in the force.

\section{Acknowledgments}

This work was supported by the Natural Science Foundation of Shanxi Province, China (Grant no. 2013011022-6 and no. 2014011006-2).

\section{References}

[1] Huang ZH. Research on fatigue life of the casting crane's girder [J]. Advanced Materials Research, 2011, 295-297: 598-602.

[2] Xie YM. Anti-fatigue Performance Analysis on Steel Crane Beam [J]. Sensors \& Transducers, 2013, 21 (5): 73-77.

[3] Kim Tae-Gu, Lee Seong-Beom, Lee Hong-Chul. A case study on engineering failure analysis of link chain [J]. Safety and health at work, 2010, 1 (1): 43-50. 
[4] Meng D, Li GF, Tan DW, Yang SL. Finite Element Analysis on Crane Girder with Variable Cross Sections Based on ANSYS [J]. Sensors \& Transducers, 2013, 21 (5): 89-94.

[5] A. Rahbar-Ranji. Dynamic Magnification Factor in a Box-Shape Steel Girder [J]. Journal of the Institution of Engineers (India): Series C, 2014, 95 (1): 11-18.

[6] You JF, Zhong YY. Application of sub-model method for missile structure analysis [J]. Guti Huojian Jishu/Journal of Solid Rocket Technology, 2014,37(2):253-257.

[7] Zhang JQ, Liu YH, Lu GZ. FEM elaborate analysis of slotted liner based on sub model method.[J] Yingyong Jichu yu Gongcheng Kexue Xuebao/Journal of Basic Science and Engineering.2005,13(4):430-435.

[8] YX Qin, WT Xie, HP Ren, X Li. Crane hook stress analysis upon boundary interpolated reproducing kernel particle method
[J]. Engineering Analysis with Boundary Elements, 2016,63:74-81.

[9] Wu Z, Lu K, Qiu H. Finite element comparative analysis of two doorframe structures in container crane [J]. 2011 Second International Conference on Mechanic Automation \& Control Engineering, MACE 2011 - Proceedings, 2011, 17 (3): 302-305.

[10] S. D. Gatsman, B. T. Gorbulinskij, A. A. Zhukov, A. A. Filatov. Improvement in durability of members of bridge cranes and sub-crane structures by decreasing the dynamic loads [J]. Metallurg, 1998, 26 (2): 46-47.

[11] U Bugaric, D Petrovic. Modeling and simulation of specialized river terminals for bulk cargo unloading with modeling of the elementary sub-systems [J]. Systems Analysis Modelling Simulation, 2002, 42 (10): 1445-1482. 